Draft VERSION July 8, 2021

Typeset using LATEX twocolumn style in AASTeX63

\title{
Effect of Dust Rotational Disruption by Radiative Torques and Implications for F-corona decrease revealed by the Parker Solar Probe
}

\author{
Thiem Hoang, ${ }^{1,2}$ Alex Lazarian, ${ }^{3,1}$ Hyeseung Lee, ${ }^{1}$ Kyungsuk Cho, ${ }^{1,2}$ Pin-Gao Gu, ${ }^{4}$ Chi-Hang NG, ${ }^{4,5}$ \\ ${ }^{1}$ Korea Astronomy and Space Science Institute, Daejeon 34055, Republic of Korea \\ ${ }^{2}$ University of Science and Technology, Korea, (UST), 217 Gajeong-ro Yuseong-gu, Daejeon 34113, Republic of Korea \\ ${ }^{3}$ Department of Astronomy, University of Wisconsin-Madison, USA \\ ${ }^{4}$ Institute of Astronomy and Astrophysics, Academia Sinica, 10617 Taipei, Taiwan \\ ${ }^{5}$ Department of Physics, National Taiwan University, 10617 Taipei, Taiwan
}

\begin{abstract}
The first-year results from the Parker Solar Probe (PSP) reveal a gradual decrease of F-coronal dust from distances of $D=0.166-0.336 \mathrm{AU}$ (or the inner elongations of $\sim 9.22-18.69 R_{\odot}$ ) to the Sun (Howard et al. 2019). Such a F-corona decrease cannot be explained by the dust sublimation scenario of the popular silicate composition that implies a dust-free-zone of boundary at heliocentric radius $R \lesssim 4-5 R_{\odot}$, but may be explained by appealing to various dust compositions with different sublimation fronts. In this paper, we present an additional explanation for the F-corona decrease using our newly introduced mechanism of dust destruction so-called Radiative Torque Disruption (RATD) mechanism. We demonstrate that RATD rapidly breaks large grains into nanoparticles so that they can be efficiently destroyed by nonthermal sputtering induced by bombardment of energetic protons from slow solar winds, which extends the dust-free-zone established by thermal sublimation to $R_{d f z} \sim 8 R_{\odot}$. Beyond this extended dust-free-zone, we find that the dust mass decreases gradually from $R \sim 42 R_{\odot}$ toward the Sun due to partial removal of nanodust by nonthermal sputtering. The joint effect of RATD and nonthermal sputtering can successfully reproduce the gradual decrease of the F-corona between $19-9 R_{\odot}$ observed by the PSP. Finally, the RATD mechanism can efficiently produce nanoparticles usually observed in the inner solar system.
\end{abstract}

Keywords: dust, solar system, corona

\section{INTRODUCTION}

Dust grains orbiting the Sun gradually spiral inward due to the loss of their angular momentum via the Poynting-Robertson (P-R) drag. When dust grains are sufficiently close to the Sun, they are sublimated because the grain temperature can exceed the sublimation threshold, producing a dust-free zone around the Sun (Russell 1929).

Mukai \& Yamamoto (1979) quantified the radius of dust-free-zone by studying dust sublimation of various dust compositions and found that dust grains are completely evaporated within a heliocentric distance of $4 R_{\odot}$ (see also Kimura \& Mann 1998 for a review). An exact location of dust-free-zone depends on grain compositions and grain sizes (see, e.g., Kobayashi et al. 2012).

Significant efforts have been made to observe the dustfree-zone from the ground during the solar eclipse (Lamy et al. 1992) and from space (e.g., Leinert et al. 1978). Near-infrared observations by Lamy et al. (1992) found no evidence of the predicted dust-free-zone, but other near-infrared observations reported the clear detection of the circumsolar dust ring at the outer edge of the dust-free zones (see Kimura \& Mann 1998 and references therein). First-year results from first two perihelion passes by the Parker Solar Probe (PSP) spacecraft, at heliocentric distances of $D=0.166-0.336 \mathrm{AU}$ from the Sun, reveal the decrease in the intensity of scattered light by circumsolar dust, so-called F-corona at small elongations $\left(\epsilon \sim 15-20^{\circ}\right.$ ) (Howard et al. 2019). The corresponding elongation in solar radii is $R_{\epsilon} \sim(0.166-0.336) \sin \left(15^{\circ}\right) \mathrm{AU} \sim 9-19 R_{\odot}$, which is suggested to see the outer edge of dust free zone (Kimura private communication). Extrapolation of the thinning trend of the F-corona suggests the possibility of observing the true dust-free-zone in the sixth flyby. In the next few years, the PSP will come closer to the Sun and reach as close as $D \sim 10 R_{\odot}\left(R_{\epsilon} \sim 2.6 R_{\odot}\right)$, which will shed light on the dust-free-zone (Fox et al. 2016). 
The gradual thinning out of the F-corona observed by the PSP between elongations of $R_{\epsilon} \sim 19-9 R_{\odot}$ is difficult to explain with the sublimation model of the standard interstellar dust that implies a sharp radius at $\sim 4-5 R_{\odot}$ (see Mann 1992). However, it could be explained by appealing to the dependence of the sublimation zone on the composition of dust particles (e.g., see Mann et al. 2004). For instance, magnesium-rich pyroxene grains are expected to sublime around $5-6 R_{\odot}$, while magnesium-rich olivine grains suffer thermal sublimation already at $\sim 10-16 R_{\odot}$ (see Kimura et al. 2002). Iron-rich olivine grains most likely sublime at even larger heliocentric distances, owing to their high absorptivity in the visible wavelength range. Sublimation of metallic grains is expected to start at $\sim 25 R_{\odot}$ (Lamy 1974).

In this paper, we aim to present an additional explanation for the F-corona decrease by considering the new effect of RAdiative Torque Disruption (RATD), which is discovered by Hoang et al. (2019) (see also Hoang 2019). The RATD mechanism is based on the fact that dust grains of irregular shapes exposed to anisotropic radiation field experience Radiative Torques (RATs; Dolginov \& Mitrofanov 1976; Draine \& Weingartner 1996; Lazarian \& Hoang 2007a; Hoang \& Lazarian 2008). RATs can spin up the grain to suprathermal rotation (Draine \& Weingartner 1996; Abbas et al. 2004) such that the resulting centrifugal stress can exceed the maximum tensile strength of the grain material, which breaks the grain into small fragments (Hoang et al. 2019). We expect that RATD rapidly breaks micron-sized grains into nanoparticles, such that the resulting nanoparticles can be destroyed rapidly by nonthermal sputtering (e.g., Jones et al. 1994) due to energetic protons of the solar wind. Indeed, the effect of RATD on enhanced thermal sublimation of icy grains was first explored in Hoang \& Tram (2020) in star-forming regions. The similar effect is expected for dust grains in the circumsolar region. Note that, previously, Paddack (1969) suggested rotational bursting of interplanetary dust by radiative torques, but his treatment is based on a rough estimate using radiation pressure and did not study the dependence on the radiation wavelength, grain shape, grain size, and composition. Our study here is based on RATs which are numerically calculated from a large sample of grain shapes and compositions and radiation wavelengths using DDSCAT (Draine \& Flatau 1994; Draine \& Weingartner 1996) and T-matrix codes (Herranen et al. 2019, 2021). We also take consider rotational damping by gas and infrared emission, which is ignored in Paddack (1969).

The structure of this paper is as follows. In Section 2, we study the disruption of dust grains into nanoparti- cles by RATD induced by solar radiation and calculate the maximum grain size as a function of the heliocentric distance. In Section 3, we study the destruction of nanoparticles by nonthermal sputtering induced by the solar wind and calculate the mass loss of dust due to sputtering. We discuss our results for observations and in-situ measurements by the PSP in Section 4. A short summary of our main findings is presented in Section 5 .

\section{ROTATIONAL DISRUPTION OF DUST GRAINS BY RADIATIVE TORQUES}

In this section, we will use the RATD mechanism to determine the upper cutoff of the grain size distribution in the F-corona.

\subsection{Basic Theory of Rotational Disruption}

A dust grain of radius a rotating at velocity $\omega$ develops a centrifugal stress due to centrifugal force, which scales as $S=\rho a^{2} \omega^{2} / 4$ with $\rho$ being the mass density of grain material (Hoang et al. 2019). When the rotation rate increases to a critical limit such that the tensile stress induced by centrifugal force exceeds the maximum tensile stress, the so-called tensile strength of the material $\left(S_{\max }\right)$, the grain is disrupted instantaneously. The critical angular velocity for the disruption is given by

$$
\begin{aligned}
\omega_{\text {cri }} & =\frac{2}{a}\left(\frac{S_{\max }}{\rho}\right)^{1 / 2} \\
& \simeq 3.6 \times 10^{9} a_{-5}^{-1} \hat{\rho}^{-1 / 2} S_{\max , 9}^{1 / 2} \mathrm{rad} / \mathrm{s},
\end{aligned}
$$

where $a_{-5}=a /\left(10^{-5} \mathrm{~cm}\right), \hat{\rho}=\rho /\left(3 \mathrm{~g} \mathrm{~cm}^{-3}\right), S_{\max }$ is the tensile strength of dust material and $S_{\max , 9}=$ $S_{\max } /\left(10^{9} \mathrm{erg} \mathrm{cm}^{-3}\right)$ is the tensile strength in units of $10^{9} \mathrm{erg} \mathrm{cm}^{-3}$.

The exact value of $S_{\max }$ depends on the dust grain composition and structure. Compact grains have higher $S_{\max }$ than porous/composite grains. Ideal material without impurity, such as diamond, can have $S_{\max } \geq$ $10^{11} \mathrm{erg} \mathrm{cm}^{-3}$ (Burke \& Silk 1974; see Hoang et al. 2019 for more details), while dust aggregates have lower tensile strength (see Tatsuuma et al. 2019; Kimura et al. 2020 for more details). In the following, we consider a range of the tensile strength from $S_{\max }=$ $10^{8}-10^{11} \mathrm{erg} \mathrm{cm}^{-3}$. The largest $S_{\max }$ is expected for nanoparticles that are likely to have compact structures.

\subsection{Grain Rotation Rate induced by Solar radiation}

Let $u_{\lambda}$ be the spectral energy density of radiation field at wavelength $\lambda$. To describe the strength of a radiation field, let define $U=u_{\mathrm{rad}} / u_{\mathrm{ISRF}}$ with $u_{\mathrm{ISRF}}=$ $8.64 \times 10^{-13} \mathrm{erg} \mathrm{cm}^{-3}$ being the energy density of the average interstellar radiation field (ISRF) in the solar neighborhood as given by Mathis et al. (1983). 
The radiation energy density at distance $R$ from the Sun is given by

$$
\begin{aligned}
u_{\text {rad }} & =\frac{L_{\star}}{4 \pi R^{2} c} \\
& \simeq 4.5 \times 10^{-5}\left(\frac{L_{\star}}{L_{\odot}}\right)\left(\frac{R}{1 \mathrm{AU}}\right)^{-2} \mathrm{erg} \mathrm{cm}^{-3},
\end{aligned}
$$

where $L_{\star}=L_{\odot}$ for the solar bolometric luminosity.

The radiation strength at distance $R$ becomes

$$
U(R) \simeq U_{0}\left(\frac{L_{\star}}{L_{\odot}}\right)\left(\frac{R}{1 \mathrm{AU}}\right)^{-2},
$$

where $U_{0}=5.2 \times 10^{7}$ is the radiation strength at $R_{0}=$ $1 \mathrm{AU}$.

The mean wavelength of the solar radiation field is defined as

$$
\bar{\lambda}=\frac{\int u_{\lambda} \lambda d \lambda}{\int u_{\lambda} d \lambda}
$$

which yields $\bar{\lambda} \approx 0.91 \mu \mathrm{m}$ for the solar-type star.

Grains subject to the anisotropic radiation field experience RATs which act to spin-up the grains to suprathermal rotation (Dolginov \& Mitrofanov 1976; Draine \& Weingartner 1996; Lazarian \& Hoang 2007a; Hoang \& Lazarian 2008). At the same time, grain rotation experiences damping due to collisions with gas species (of proton density $n_{\mathrm{H}}$ and temperature $T_{\text {gas }}$ ) and infrared emission (see Appendix C). The grain rotation velocity induced by RATs is given by (Hoang et al. 2019 and Hoang 2019)

$$
\begin{aligned}
\omega_{\mathrm{RAT}} & \simeq 3.2 \times 10^{8} \gamma a_{-5}^{0.7} \bar{\lambda}_{0.5}^{-1.7} \\
& \times\left(\frac{U}{\hat{n} \hat{T}_{\text {gas }}^{1 / 2}}\right)\left(\frac{1}{1+F_{\mathrm{IR}}}\right) \mathrm{rad} \mathrm{s}^{-1},
\end{aligned}
$$

for grains with $a \lesssim a_{\text {trans }}$, and

$$
\begin{aligned}
\omega_{\mathrm{RAT}} \simeq & 1.6 \times 10^{9} \frac{\gamma}{a_{-5}^{2}} \bar{\lambda}_{0.5} \\
& \times\left(\frac{U}{\hat{n} \hat{T}_{\mathrm{gas}}^{1 / 2}}\right)\left(\frac{1}{1+F_{\mathrm{IR}}}\right) \mathrm{rad} \mathrm{s}^{-1},
\end{aligned}
$$

for grains with $a>a_{\text {trans }}$ where $a_{\text {trans }} \sim \bar{\lambda} / 1.8$ is the transition grain size where RAT efficiency $Q_{\Gamma}$ changes the slope from $\eta \sim-3$ to $\eta=0$ (Lazarian \& Hoang 2007a; see Appendix C).

In the above equations, $\bar{\lambda}_{0.5}=\bar{\lambda} / 0.5 \mu \mathrm{m}, \hat{n}=$ $n_{H} / 10 \mathrm{~cm}^{-3}, \hat{T}_{\text {gas }}=T_{\text {gas }} / 100 \mathrm{~K}, F_{\mathrm{IR}}$ is the dimensionless parameter that describes the grain rotational damping by infrared emission. In addition to gas and IR damping, grains experience rotational damping due to plasma drag and ion collisions (Draine \& Lazarian 1998; Hoang et al. 2010). However, subject to intense solar radiation field, the infrared damping is dominant $\left(F_{\mathrm{IR}} \gg 1\right)$, such that the grain rotation rate does not depend on the local gas properties (see Appendix $\mathrm{C}$ for more details).

\subsection{Grain disruption size and time vs. heliocentric distance}

For strong solar radiation fields considered in this paper, $F_{\mathrm{IR}} \gg 1$, from Equations (5) and (1), one can obtain the disruption grain size:

$$
a_{\text {disr }} \simeq 0.18\left(\gamma^{-1} U^{-1 / 3} \bar{\lambda}_{0.5}^{1.7} S_{\max , 9}^{1 / 2}\right)^{1 / 2.7} \mu \mathrm{m},
$$

for $a_{\text {disr }} \leq a_{\text {trans }}$.

At $R=1 \mathrm{AU}$, one obtains $a_{\text {disr }} \sim 0.02 \mu \mathrm{m}$ for the typical parameters of $S_{\max , 9}=1, \gamma=1$, and $\lambda=0.91 \mu \mathrm{m}$. At $R=0.1 \mathrm{AU}\left(21 R_{\odot}\right.$, one has $a_{\mathrm{disr}}=0.011 \mu \mathrm{m}$. At $R=0.05 \mathrm{AU}\left(10 R_{\odot}\right), a_{\mathrm{disr}} \sim 0.0096 \mu \mathrm{m}$. At $R=$ $0.02 \mathrm{AU}\left(4 R_{\odot}\right), a_{\text {disr }}=0.0076 \mu \mathrm{m}$. So, within $R=$ $0.5 \mathrm{AU}$, nanoparticles of size $a<a_{\mathrm{disr}} / 2 \sim 0.0055 \mu \mathrm{m}$ $(5.5 \mathrm{~nm})$ are abundant due to RATD.

Due to the decrease of $\omega_{\mathrm{RAT}}$ with the grain size for $a>a_{\text {trans }}$ (see Eq. 5), very large grains would not be disrupted. The maximum size of grains that can still be disrupted by RATD is given by (Hoang 2019)

$$
\begin{aligned}
a_{\text {disr }, \text { max }} \simeq & 9.6 \gamma \bar{\lambda}_{0.5}\left(\frac{U}{\hat{n} \hat{T}_{\text {gas }}^{1 / 2}}\right)^{1 / 2}\left(\frac{1}{1+F_{\mathrm{IR}}}\right) \\
& \times \rho S_{\text {max }, 9}^{-1 / 2} \mu \mathrm{m} .
\end{aligned}
$$

Figure 1 (upper panel) shows the grain disruption size vs. the heliocentric distance for the different tensile strength. For $R<5 R_{\odot}(0.24 \mathrm{AU})$, only very small grains of sizes $a<0.005 \mu \mathrm{m}(5 \mathrm{~nm})$ can survive against RATD, assuming compact grains of $S_{\max } \sim 10^{9} \mathrm{erg} \mathrm{cm}^{-3}$. Ideal nanoparticles with $S_{\max } \sim$ $10^{11} \mathrm{erg} \mathrm{cm}^{-3}$ can have a maximum size of $a<0.03 \mu \mathrm{m}$ $(30 \mathrm{~nm})$. For $R<50 R_{\odot}(0.5 \mathrm{AU})$, we predict only small grains of $a<0.025 \mu \mathrm{m}(25 \mathrm{~nm})$.

The characteristic timescale for rotational desorption can be estimated as (Hoang et al. 2019):

$$
\begin{aligned}
t_{\mathrm{disr}, 0} & =\frac{I \omega_{\mathrm{disr}}}{d J / d t}=\frac{I \omega_{\mathrm{disr}}}{\Gamma_{\mathrm{RAT}}} \\
& \simeq 36.5\left(\gamma U_{7}\right)^{-1} \bar{\lambda}_{0.5}^{1.7} \hat{\rho}^{1 / 2} S_{\max , 9}^{1 / 2} a_{-5}^{-0.7} \text { days }
\end{aligned}
$$

for $a_{\text {disr }}<a \lesssim a_{\text {trans }}$, and

$$
t_{\text {disr }, 0} \simeq 2.7\left(\gamma U_{7}\right)^{-1} \bar{\lambda}_{0.5}^{-1} \hat{\rho}^{1 / 2} S_{\max , 9}^{1 / 2} a_{-5}^{2} \text { days }
$$

for $a_{\text {trans }}<a<a_{\text {disr,max }}$ where $U_{7}=U / 10^{7}$. 
Figure 1 (lower panel) shows the disruption time obtained for the different tensile strengths. The disruption time decreases rapidly with the heliocentric distance.

Our results reveal that grains within $0.1 \mathrm{AU}\left(21 R_{\odot}\right)$ are dominated by nanoparticles of size $a<0.01 \mu \mathrm{m}(10$ $\mathrm{nm}$ ) due to RATD, as illustrated in Figure 2).

\section{DESTRUCTION OF NANOPARTICLES BY THE SOLAR WIND}

In this section, we will show that smallest nanoparticles near the Sun produced by RATD will be efficiently destroyed by bombardment of energetic protons from the solar wind.

\subsection{Solar wind components}

The solar wind usually consists of very slow $(v \sim$ $\left.200-300 \mathrm{kms}^{-1}\right)$, slow $\left(v \sim 300-700 \mathrm{kms}^{-1}\right)$, and fast $\left(v>700 \mathrm{~km} \mathrm{~s}^{-1}\right)$ solar winds (see, e.g., Mukai \& Schwehm (1981)). The very slow winds (VSLW) are observed frequently in the inner solar region of heliocentric distance $R<1$ AU such as by Helios $1 / 2$, but are rarely near the Earth and the density of VSLW is much higher than that of fast solar winds (Sanchez-Diaz et al. 2016).

The number density of solar wind protons varies with the heliocentric distance and is given by

$$
n_{p}(R)=n_{0}\left(\frac{R}{R_{0}}\right)^{-2}
$$

where $n_{0}=6 \mathrm{~cm}^{-3}$ at $R_{0}=1 \mathrm{AU}$ (see e.g., Venzmer \& Bothmer (2018)). At heliodistance of $R=0.1 \mathrm{AU}(\sim$ $\left.21 R_{\odot}\right)$, the proton density increases to $n_{p}=600 \mathrm{~cm}^{-3}$.

\section{2. $\quad$ Nonthermal sputtering}

Bombardment of energetic protons gradually erodes the grain surface via the sputtering mechanism (see e.g., Hoang et al. 2015; Hoang \& Lee 2020).

To calculate the sputtering time, we assume a uniform distribution of proton velocity, and the mean velocity of solar wind (sw) protons is $\bar{v}_{\mathrm{sw}} \sim 400 \mathrm{~km} \mathrm{~s}^{-1}$. Let $Y_{\mathrm{sp}}$ be the sputtering yield induced by the proton bombardment. The characteristic timescale, $t_{\mathrm{nsp}}$, of grain destruction by nonthermal sputtering for a spherical grain of size $a$ is defined by

$$
\begin{aligned}
t_{\mathrm{nsp}} & =\frac{a}{d a / d t}=\frac{4 \rho a}{n_{p} m_{p} \bar{v}_{\mathrm{sw}} Y_{\mathrm{sp}} \bar{A}_{\mathrm{sp}}} \\
& \simeq 3486\left(\frac{\hat{\rho}}{n_{2} v_{2}}\right)\left(\frac{12}{\bar{A}_{\mathrm{sp}}}\right)\left(\frac{a}{10^{-7} \mathrm{~cm}}\right)\left(\frac{0.5}{Y_{\mathrm{sp}}}\right) \operatorname{days}(12)
\end{aligned}
$$

where $n_{2}=n_{\mathrm{p}} /\left(100 \mathrm{~cm}^{-3}\right)$, and $\bar{A}_{s p}$ is the mean atomic mass of sputtered atoms from the grain (see e.g., Hoang \& Lee 2020).
At $R \sim 0.1 \mathrm{AU}\left(\sim 21 R_{\odot}\right), n_{p} \sim 600 \mathrm{~cm}^{-3}$ (see Eq. 11), the sputtering time is $t_{\mathrm{nsp}}=1093\left(a / 10^{-7} \mathrm{~cm}\right)$ days, assuming $Y_{\mathrm{sp}}=0.5$. Thus, the sputtering time is much longer than the rotational disruption time $t_{\text {disr }}$ for grains of $a>a_{\text {dirs }}$ (see the right panel of Figure 1).

To study if nonthermal sputtering can be fast enough to destroy nanoparticles before they are dragged to the sublimation zone by the Poynting-Robertson (P-R) drag, we calculate the ratio of nonthermal sputtering time to the P-R drag time (see Eq. B5):

$$
\begin{aligned}
\frac{t_{\mathrm{nsp}}}{t_{P-R}} \approx & 0.03\left(\frac{\left\langle Q_{\mathrm{pr}}\right\rangle}{0.1}\right)\left(\frac{500 \mathrm{~cm}^{-3}}{n_{p}}\right)\left(\frac{300 \mathrm{~km} \mathrm{~s}^{-1}}{\bar{v}_{\mathrm{sw}}}\right) \times \\
& \times\left(\frac{0.5}{Y_{\mathrm{sp}}}\right)\left(\frac{\mathrm{AU}}{R}\right)^{2} .
\end{aligned}
$$

where $\left\langle Q_{\mathrm{pr}}\right\rangle$ is the averaged radiation pressure crosssection efficiency which is a function of the grain size.

For nanoparticles in the solar radiation field with $\left\langle Q_{\text {pr }}\right\rangle \lesssim 0.1$ (see Figure 7), Equation (13) reveals that nanoparticles are rapidly destroyed by nonthermal sputtering before dragged into the sublimation zone by the $\mathrm{P}-\mathrm{R}$ drag. The efficiency of nonthermal sputtering is increased with decreasing the heliocentric distance due to the increase of proton density (see Equation 11).

We note that for nanoparticles, rotational disruption by stochastic mechanical torques by the solar wind is less efficient than nonthermal sputtering because energetic protons tend to pass through the grain (Hoang \& Lee 2020). Misconi (1993) studied the rotational bursting of circumsolar dust caused by solar winds protons using the "Paddack effect"-"windmill" (Paddack \& Rhee 1975). However, Misconi (1993) did not take into account the effect of proton passage and assumed a rather low tensile strength of $S_{\max }=10^{6} \mathrm{erg} \mathrm{cm}^{-3}$ for circumsolar dust, which is quite low for dust grains of compact structures. The rotational disruption by regular mechanical torques due to the interaction of the solar wind with irregular grains (Lazarian \& Hoang 2007b; Hoang et al. 2018) is expected to be more efficient. A detailed study of this effect is presented elsewhere.

\subsection{Extended dust-free-zone and decrease of dust mass due to sputtering}

Figure 3 compares the nonthermal sputtering time evaluated at $a=a_{\text {disr }}$ and $a=a_{\text {disr }} / 2$ to other relevant timescales. The sputtering yield for protons of $\bar{v}_{\mathrm{sw}}$ is calculated as in Hoang \& Lee (2020). The P-R drag time is calculated using Equation (B5) where $\left\langle Q_{\mathrm{pr}}\right\rangle$ is a function of the grain size as shown in Figure 7 . The sublimation time $t_{\text {sub }}$ for two grain sizes at $T_{d}=1500 \mathrm{~K}$ is shown for comparison (see Eq. B7). 

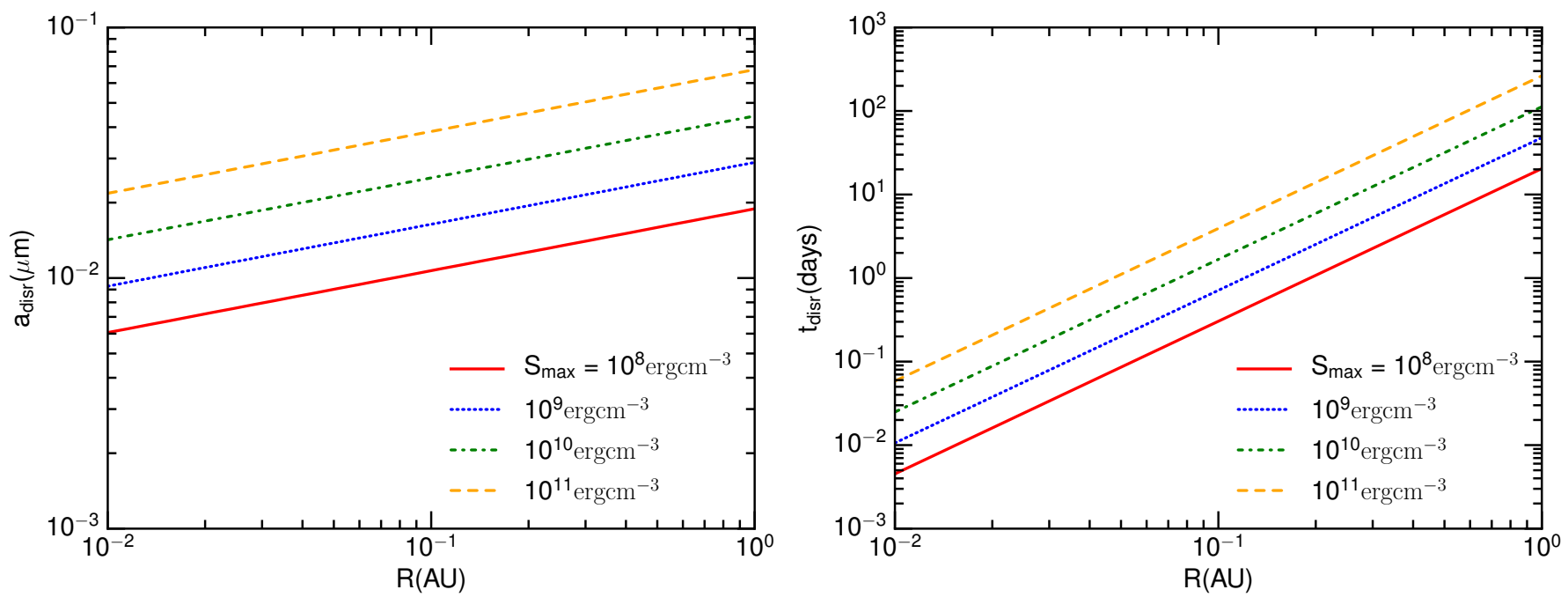

Figure 1. Left panel: Grain disruption size, $a_{\mathrm{disr}}$, as a function of the heliocentric distance for the different tensile strengths. Disruption size is smaller for weaker grains (i.e., lower $S_{\max }$ ). Right panel: disruption time evaluated at $a=a_{\text {disr }}$. Large grains of $a>0.01 \mu \mathrm{m}$ are disrupted rapidly on a timescale of $t_{\text {disr }}<10$ days.

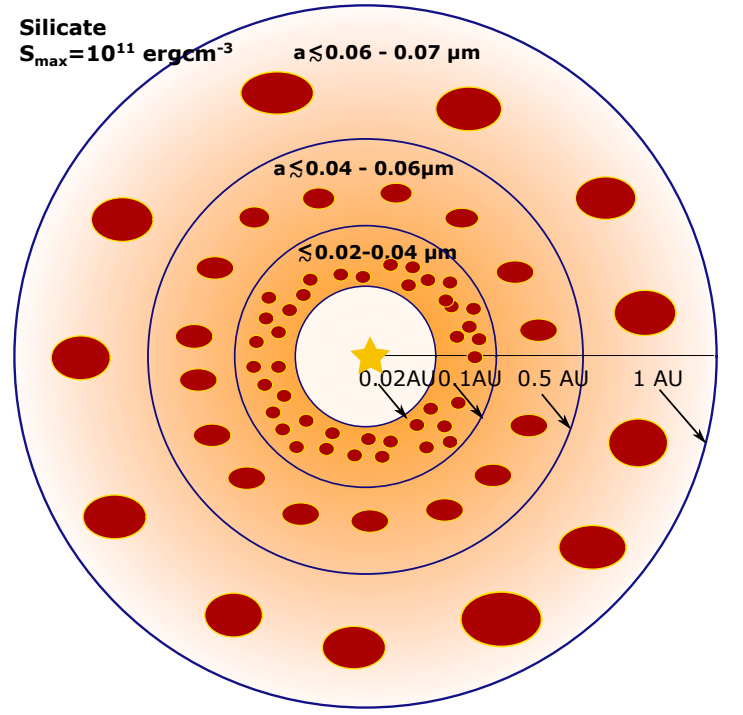

Figure 2. Schematic illustration of spatial variation of grain sizes in the F-corona as a result of RATD: abundance of small grains increases toward the Sun.

The dashed vertical lines denote the location of the extended dust-free-zone determined by nonthermal sputtering $R_{\mathrm{sp}}$. The sputtering radius is $R_{\mathrm{sp}} \sim 7-13 R_{\odot}$ for grains of $S_{\max } \sim 10^{9}-10^{11} \mathrm{erg} \mathrm{cm}^{-3}$ for silicate and $8 R_{\odot}$ for $S_{\max } \sim 10^{9} \mathrm{erg} \mathrm{cm}^{-3}$ for graphite. Note that graphite is not found in the interplanetary dust, the choice of graphite here is an example of a highly absorptive dust, such as hydrogenated amorphous carbon.

In Figure 3 , the region where $t_{\mathrm{nsp}}\left(a=a_{\text {disr }}\right) \lesssim$ $t_{\mathrm{sub}}\left(T_{d}=1500 \mathrm{~K}\right)$ is considered an extended dust-freezone. Note that the classical dust-free-zone is defined by thermal sublimation where grains are destroyed rapidly on a timescale of $t_{\mathrm{sub}}\left(T_{d}=1500 \mathrm{~K}\right)$. Thus, if nonthermal sputtering could destroy grains in the same time beyond this zone, then, the dust-free-zone is extended.

Beyond the extended dust-free-zone, nanoparticles of $a<a_{\text {disr }}$ can be depleted by nonthermal sputtering because $t_{\mathrm{nsp}}(a)<t_{\mathrm{sub}}$. As a result, the mass loss of dust via nonthermal sputtering is decreasing outward, or the total dust mass decreases inward until the dustfree-zone.

Let $a_{\text {nsp }}$ be the critical size of nanoparticles that are destroyed by nonthermal sputtering. One can determine $a_{\text {nsp }}$ by setting $t_{\text {nsp }}\left(a_{\text {nsp }}\right)=t_{\text {sub }}$. All grains smaller than $a_{\text {nsp }}$ are destroyed by nonthermal sputtering. The value of $a_{\text {nsp }}$ will increase with decreasing $R$ due to the increase of the proton density (see Eq. 11).

Figure 4 illustrates the grain size distribution $\left(d n / d a \propto a^{-3.5}\right)$ as a result of the RATD mechanism and nonthermal sputtering. Only small grains within a narrow range (shaded area) could survive in the Fcorona.

Let $f_{\text {high-J }}$ be the fraction of grains that can be spunup to suprathermal rotation and then be disrupted by RATD (see more in Section 4. Then, we can calculate the decrease of the dust mass as a function of heliocentric distance as follows:

$$
\begin{aligned}
\frac{M_{d}(R)}{M_{d}(R, 0)}= & \frac{\int_{a_{\mathrm{nsp}}}^{a_{\mathrm{nisr}}} f_{\mathrm{high}-\mathrm{J}}\left(4 \pi a^{3} \rho / 3\right)(d n / d a) d a}{M_{d}(R, 0)} \\
& +\frac{\left(1-f_{\mathrm{high}-\mathrm{J}}\right) M_{d}(0)}{M_{d}(0)}
\end{aligned}
$$



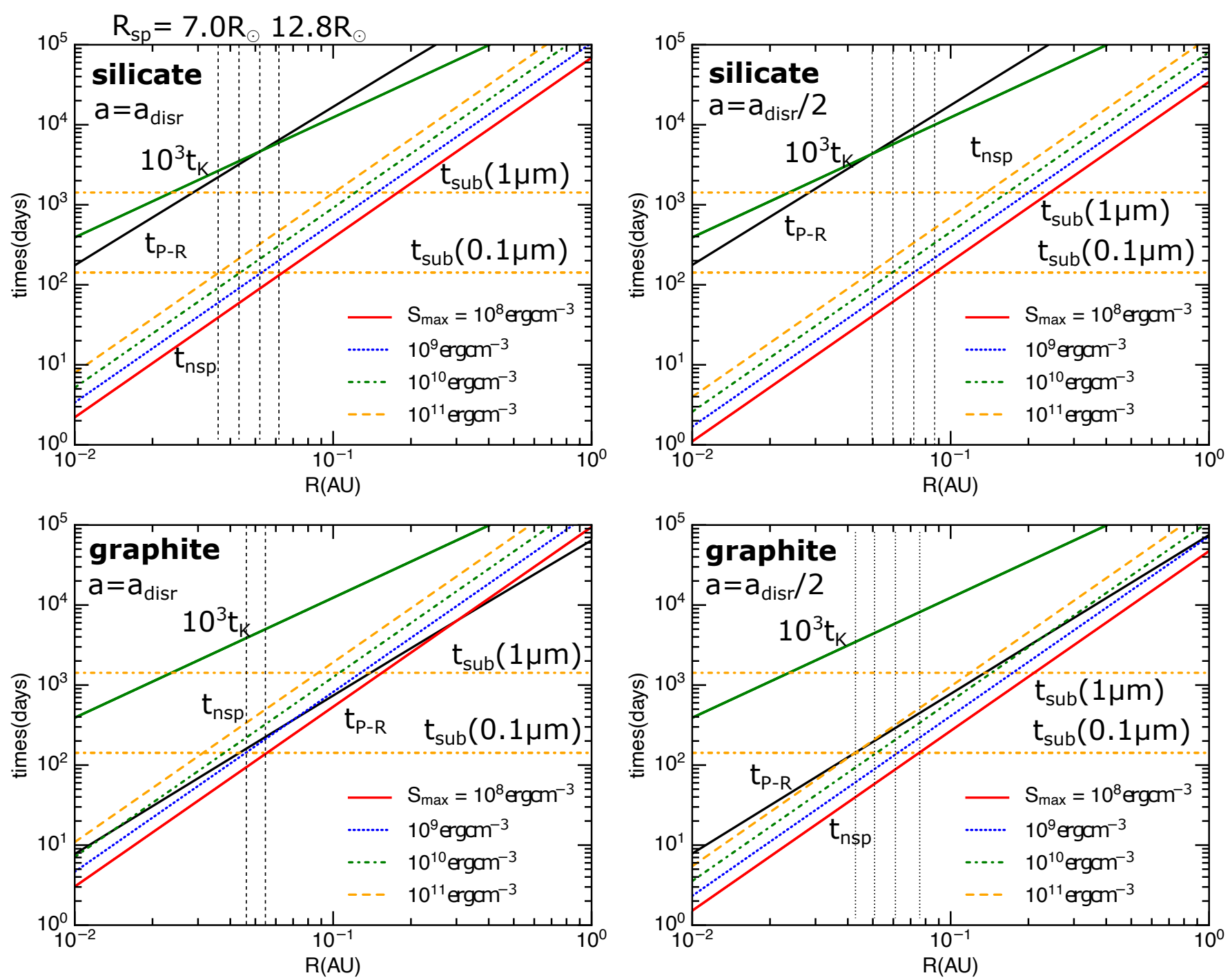

Figure 3. Comparison of the characteristic timescales due to various processes, including nonthermal sputtering $\left(t_{\mathrm{nsp}}\right)$, the P-R drag $\left(t_{P-R}\right)$, Keplerian time $t_{K}$ for grains of sizes $a=a_{\text {disr }}$ and various $S_{\max }$ (left panels). The sublimation time $\left(t_{\text {sub }}\right)$ for classical grains $a=0.1 \mu \mathrm{m}$ and $1 \mu \mathrm{m}$ are shown for comparison. Right panels show results for $a=a_{\text {disr }} / 2$. The dashed vertical lines denote the location where $t_{\mathrm{nsp}}=t_{\mathrm{sub}}(a=0.1 \mu \mathrm{m})$.

where $M_{d}(R, 0)$ is the original dust mass in the absence of sputtering

$$
M_{d}(R, 0)=\int_{a_{\min }}^{a_{\max }}\left(\frac{4 \pi a^{3} \rho}{3}\right)\left(\frac{d n}{d a}\right) d a
$$

where a power-law grain size distribution of $d n / d a=$ $C a^{-3.5}$ with $a_{\min }=3 \AA$ is adopted.

Figure 5 shows the variation of the relative dust mass $M_{d} / M_{d}(0)$ vs. the heliocentric distance as a result of RATD and sputtering for the different tensile strength and perfect disruption with $f_{\text {high }-\mathrm{J}}=1$. We see that $M_{d} / M_{d}(0)$ starts to decrease considerably from $R \sim 0.2$ AU $\left(42 R_{\odot}\right)$, and a significant mass loss occurs at $R \lesssim$ $0.03 \mathrm{AU}\left(6 R_{\odot}\right)$, which suggests an extended dust-free- zone. Grains made of weak material are more efficiently disrupted by RATD and experience larger mass loss.

Note that in the absence of RATD, nonthermal sputtering still can remove nanoparticles, but the fraction of depleted mass is negligible because the dust mass is mostly dominated by largest grains of $a \sim a_{\max }$. The effect of RATD converts large grains $a>a_{\text {disr }}$ into smaller sizes and increases the abundance of nanoparticles. As a result, the amount of dust mass depleted by sputtering is significantly enhanced. However, it is uncertain whether RATD is efficient for very large grains (VLGs) of $a>\bar{\lambda} / 0.1 \sim 9 \mu \mathrm{m}$ due to the lack of numerical calculations of RATs. If circumsolar dust is dominated by VLGs, the effect of RATD and nonthermal sputtering is less efficient. 


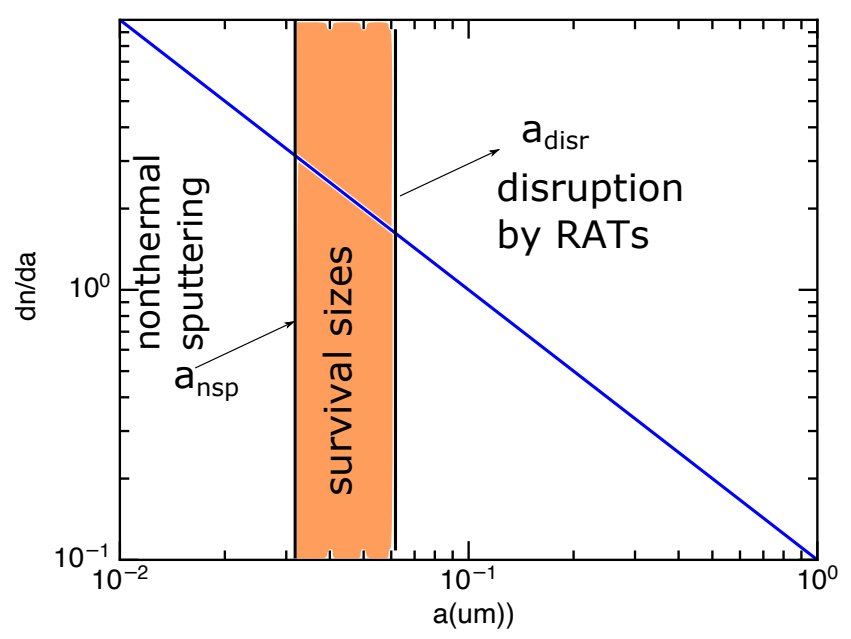

Figure 4. Grain size distribution as a result of RATD and sputtering. Grains larger than $a_{\text {disr }}$ are disrupted into grains of $a<a_{\mathrm{disr}}$, while nonthermal sputtering removes grains of $a<a_{\mathrm{nsp}}$.

\section{DISCUSSION}

\subsection{Effects of RATD and nonthermal sputtering}

We have shown that RATD rapidly breaks large dust grains into nanoparticles. This results in the increase in the abundance of small grains relative to large ones toward the Sun. Subsequently, nonthermal sputtering by the solar wind destroys smallest grains. Due to the increase of the solar wind density with decreasing the heliocentric distance, nonthermal sputtering rate is rapidly increased. As a result, dust grains can be completely removed by nonthermal sputtering near the Sun, which extends the dust-free-zone implied by thermal sublimation. We found that the extended dust-free-zone is located within a radius of $R_{d f z} \sim 6-12 R_{\odot}$ (depending on $S_{\text {max }}$, which is much larger than the classical one defined by thermal sublimation of $4-5 R_{\odot}$.

Our results also suggest that F-corona dust as well as dust in the inner solar system $(R<1 \mathrm{AU})$ mostly contain nanoparticles of size $a \sim 1-10 \mathrm{~nm}$ (see Figure 4). This is a plausible explanation for the existence of nanodust detected by in-situ measurements (see, e.g., Mann et al. 2007; Mann 2017; Ip et al. 2019). This RATD mechanism is more efficient than collisional fragmentation previously thought (see e.g., Mann et al. 2007 for discussion of various mechanisms to form nanodust in the inner solar system).

In-situ measurements by dust detector on board the Helio spacecrafts reported the F-corona decrease at heliocentric distances between $D=0.3-1 \mathrm{AU}$ (Gruen et al. 1985). This is thought due to the mutual collisions that makes grains smaller and decrease of the forward scattering cross-section. However, the RATD appears to be more efficient in producing small grains due to its short timescale.

Note that RATD is valid for grains of $\bar{\lambda} / a>0.1$. For larger grains of $a>\bar{\lambda} / 0.1 \sim 9 \mu \mathrm{m}$, RATs of such very large grains are not yet available due to the lack of numerical calculations because it requires expensive computations to achieve reliable results of RATs for grains of $2 \pi a / \lambda \gg 1$ (see e.g., Draine \& Flatau 2004). Expecting the decrease of RATs with increasing $a$, the disruption may still be important when the decrease of RATs is compensated by the increase of the radiation energy density.

\subsection{Implications for the first-year results by the Parker Solar Probe}

The first-year results from the PSP at heliocentric distances of $D=0.16-0.25 \mathrm{AU}\left(34.3-53.7 R_{\odot}\right)$ reveal the gradual decrease the F-corona (Howard et al. 2019). With the elongation $\epsilon \sim 15-20^{\circ}$, one can estimate the corresponding elongation in solar radii $R_{\epsilon} \sim$ $(0.166-0.336) \sin \left(15^{\circ}\right) \mathrm{AU} \sim 9-19 R_{\odot}$.

To explain the first-year PSP results, let us first recall that the intensity of scattered sunlight by dust depends on the dust scattering cross-section as follows:

$$
\begin{aligned}
I_{\mathrm{sca}} & \propto \sigma_{\mathrm{sca}}(\lambda)=\int Q_{\mathrm{sca}}(a, \lambda) \pi a^{2}\left(\frac{d n}{d a}\right) d a, \\
& \propto \int\left(\frac{Q_{\mathrm{sca}}(a, \lambda)}{a}\right)\left(\frac{\pi a^{3} d n}{d a} d a\right),
\end{aligned}
$$

where the term in the second bracket describes the dust mass.

Equation (16) reveals that the decrease of dust mass by sputtering and RATD as shown in Figure 5 directly reduces the observed intensity. Moreover, the decrease of grain sizes by RATD also decreases the scattering efficiency $Q_{\text {sca }}$ because in the small grain limit $(a \ll \lambda)$, $Q_{\text {sca }}(a, \lambda) \sim(2 \pi a / \lambda)^{4}$. Thus, the joint effect of RATD and sputtering would decrease the brightness of the Fcorona.

Figure 6 illustrates the F-corona as a result of RATD and nonthermal sputtering, which would be observed with the PSP. The extended dust-free-zone is located at $R=6 R_{\odot}$. Beyond this radius, the F-corona decreases with the radius, starting from $42 R_{\odot}$ to the dust-freezone.

Note that the sublimation radius given by Equation (B12) reveals the range of the dust-free-zone between $4-5 R_{\odot}$ for silicate grains of sizes $a \sim 0.1-0.001 \mu \mathrm{m}$. Therefore, even with the effect of RATD, thermal sublimation alone cannot explain the thinning-out of circumsolar dust observed from $R_{\epsilon} \lesssim 19 R_{\odot}$. However, our 

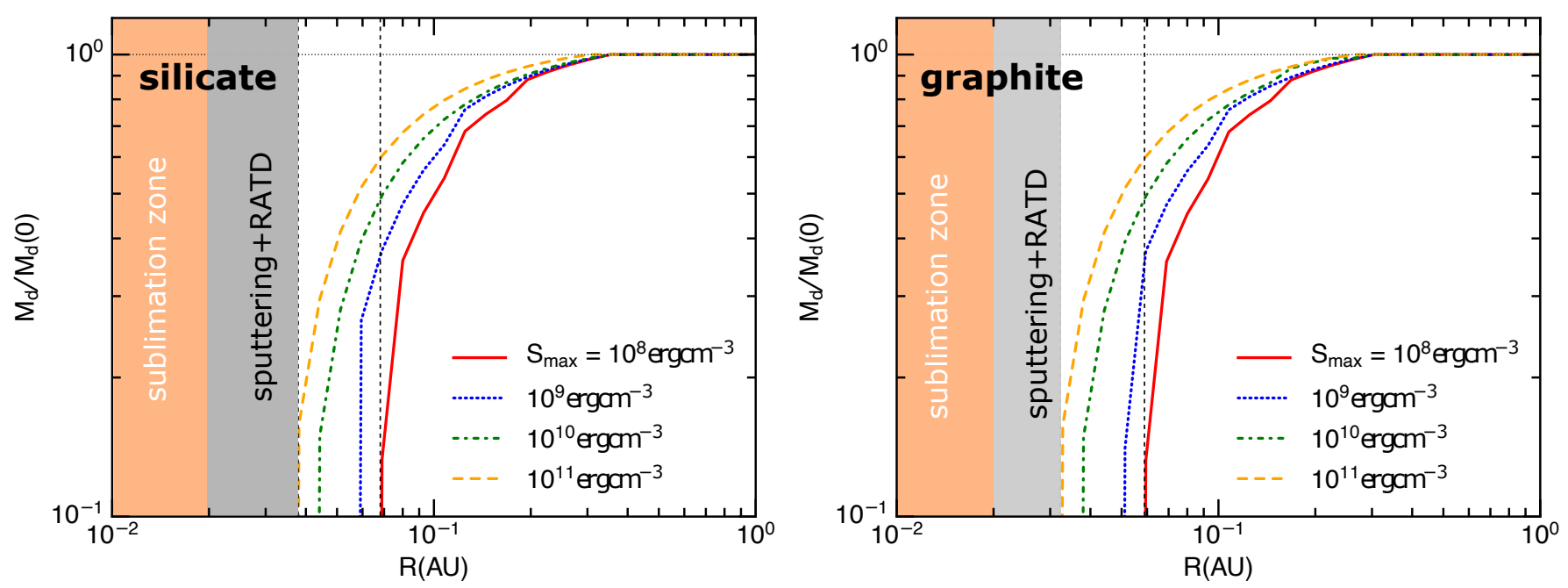

Figure 5. Decrease of the dust mass $\left(M_{d} / M_{d}(0)\right)$ with heliocentric distance due to RATD and nonthermal sputtering by the solar wind for silicate (left panel) and graphite (right panel) grains where $M_{d}(0)$ is the original dust mass. Four values of the tensile strength $S_{\max }=10^{8}-10^{11} \mathrm{erg} \mathrm{cm}^{-3}$ are considered. The dust mass decreases toward the Sun, starting from $\sim 0.2 \mathrm{AU}$ (for highest strength) and reaches the extended dust-free-zone predicted by RATD and sputtering effects (gray shaded area).

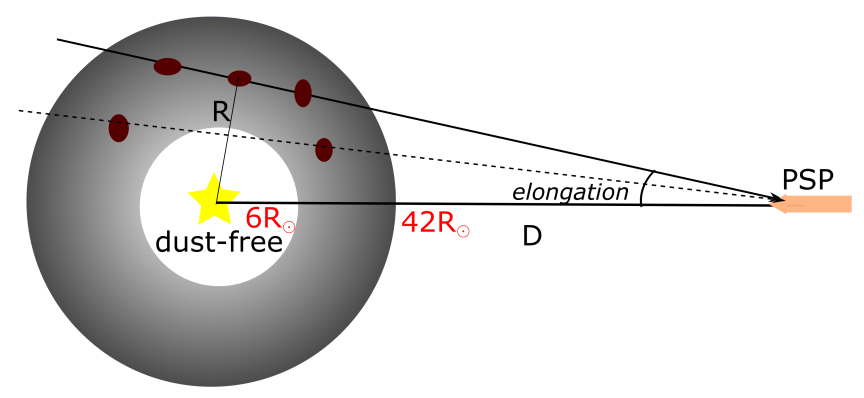

Figure 6. Schematic illustration of the F-corona predicted by RATD and nonthermal sputtering which would be observed by the PSP at heliocentric distance of $D=0.3 \mathrm{AU}$ and various elongation angles. The F-corona decrease is illustrated by a radial gradient, starting from $42 R_{\odot}$ to the edge of the extended dust-free-zone at $6 R_{\odot}$.

results shown in Figure 5) indicate that the joint effect of RATD and nonthermal sputtering could successfully explain the gradual decrease of F-corona toward the Sun. Moreover, the PSP's observation is consistent with our result with the largest value of $S_{\max }$ because this model predicts the F-corona decrease from a closest distance of $19 R_{\odot}$.

The PSP is planned to undergo 24 orbits around the Sun. The latest orbits (22-24) will reach the closest distance of $10.74 R_{\odot}$ (Fox et al. 2016). Previous studies predict that the dust-free-zone is between $4-5 R_{\odot}$, which cannot be confirmed with the PSP. We found that the joint action of RATD and sputtering increase the radius of dust-free-zone to $7-11 R_{\odot}$ (see Figure 3$)$. This would be tested with the upcoming orbits of the PSP.
Finally, it is worth to mention that the variation of the grain size distribution by RATD affects the effect of radiation pressure. Indeed, as grains become smaller, the radiation cross-section efficiency $\left\langle Q_{\mathrm{pr}}\right\rangle$ decreases with decreasing grain size (see Figure 7 ). Thus, the ratio of radiation force to gravity $\beta=F_{\text {rad }} / F_{\text {gra }}$ becomes smaller than unity (see Equation B4), such that radiation pressure cannot blow these nanoparticles away.

\subsection{Effects of grain alignment with the magnetic field vs. the radiation direction}

We have assumed that RATs spin up grains to their maximum rotational angular velocity and ignored the effect of grain alignment. Thus, our obtained results (e.g., Figure 5) are considered an upper limit of the rotational disruption effect. Previous studies show that in addition to spin up, RATs induce grain alignment along the magnetic field direction, which is usually referred to as B-RAT. Moreover, subject to the intense solar radiation, the alignment axis may change from the magnetic field (B-RAT) to the radiation direction (i.e., k-RAT; see more details in Lazarian \& Hoang 2019). As suggested by Parker (1958), the magnetic field near the Sun is almost radial. Therefore, our obtained results are weakly affected by the magnetic field because the radiation and magnetic field are nearly parallel.

We have also assumed that all aligned grains have their maximum angular momentum $\omega_{\mathrm{RAT}}$ (Eqs. 5 and (C24), which corresponds to the situation that all grains are driven to high-J attractors (Hoang \& Lazarian 2016; Hoang \& Lazarian 2008). In general, the fraction of grains on high-J attractors, denoted by $f_{\text {high }-\mathrm{J}}$, depends on the grain properties (shape, size, and magnetic prop- 
erties), and $0<f_{\text {high-J }} \leq 1$. An extensive study for numerous grain shapes and compositions by Herranen et al. (2021) found $f_{\text {high }-\mathrm{J}} \sim 0.2-0.7$, depending on the grain shapes. The presence of iron inclusions is found to increase $f_{\text {high-J }}$ to unity (Hoang \& Lazarian 2016). It is likely that for a given size, the grains with iron inclusions would be fully disrupted first. As a consequence, we may expect to have an increase of pure "iron" tiny grains or grains with significantly increased magnetic content and pure paramagnetic grains. For the latter fraction, thermal sublimation will be only the destruction effect for the fraction of grains without high-J attractors that RATD is not effective. At the same time, our predicted dependence will be applicable to the population of high-J grains. More details about the relation of alignment and the RATD are given in Lazarian \& Hoang (2021). Therefore, the decrease of dust mass predicted in this paper (Fig. 5) is an upper limit and may be lower by a factor $f_{\text {high-J }}$. As a result, the decrease of $M_{d}$ (F-corona) would start from a smaller heliodistance of $<42 R_{\odot}$.

\subsection{Existence of circumsolar dust around $4-5 R_{\odot}$ and constraining the fraction of high- $J$ attractors}

Circumsolar dust is an ideal environment to test RATD mechanism because we have both indirect and direct measurement of dust. Observations at ultraviolet, visible, and near-infrared wavelength reveal the existence of large grains between $1-10 \mu \mathrm{m}$ in the circumsolar corona around $4-5 R_{\odot}$. This implies that the RATD effect is not perfect (i.e., $f_{\text {high-J }}<1$ ), as we discussed in the previous subsection. Detailed modeling of the F-corona brightness with observations would provide a constraint on the efficiency of RATD.

\section{SUMMARY}

We provide an additional explanation for the F-corona decrease revealed by the Parker Solar Probe based on the RATD mechanism, which does not strongly depend on the grain composition but on the grain tensile strength (internal structure). Our main results are summarized as follows:

1. We find that RATD can rapidly break large dust grains into smaller fragments, resulting in an in- crease in the abundance of nanoparticles with decreasing the heliocentric distance. This RATD mechanism can explain the ubiquitous existence of nanoparticles in the inner solar system.

2. We study destruction of circumsolar dust via nonthermal sputtering due to bombardment of energetic protons from the solar wind and find that nonthermal sputtering is efficient in destroying smallest nanoparticles. The nonthermal sputtering is faster than the P-R drag because the radiation pressure cross-section efficiency is significantly decreased for nanoparticles.

3. Due to the effect of RATD and nonthermal sputtering, the dust-free-zone is extended in comparison to the sublimation zone predicted by the silicate dust.

4. Beyond the dust-free-zone, we find that nonthermal sputtering can remove smallest nanoparticles, which results in the decrease of dust mass toward the Sun. This effect can explain the gradual decrease of the F-corona observed by Parker Solar Probe between the heliocentric distances of $D=$ $0.166-0.336 \mathrm{AU}$ or elongations $R_{\epsilon} \sim 9-19 R_{\odot}$ at $\epsilon=15^{\circ}$.

\section{ACKNOWLEDGMENTS}

We thank the second anonymous referee for helpful comments that improved the presentation of our paper. TH thanks Hiroshi Kamura for helpful discussion. TH acknowledges the support by the National Research Foundation of Korea (NRF) grants funded by the Korea government (MSIT) through the Basic Science Research Program (2017R1D1A1B03035359) and Midcareer Research Program (2019R1A2C1087045). AL acknowledges the support from NSF AST 1715754 grant. KSC acknowledges the support from KASI R\&D program (2020-1-850-02). PGG acknowledges the support from MOST in Taiwan through the grant MOST 1052119-M-001-043-MY3.

\section{APPENDIX}

\section{A. RADIATION PRESSURE CROSS-SECTION}

Absorption cross-section $C_{\mathrm{abs}}$ and absorption efficiency $Q_{\mathrm{abs}}=C_{\mathrm{abs}} / \pi a^{2}$. The scattering efficiency is $Q_{\mathrm{sca}}=$ $C_{\text {sca }} /\left(\pi a^{2}\right)$. 

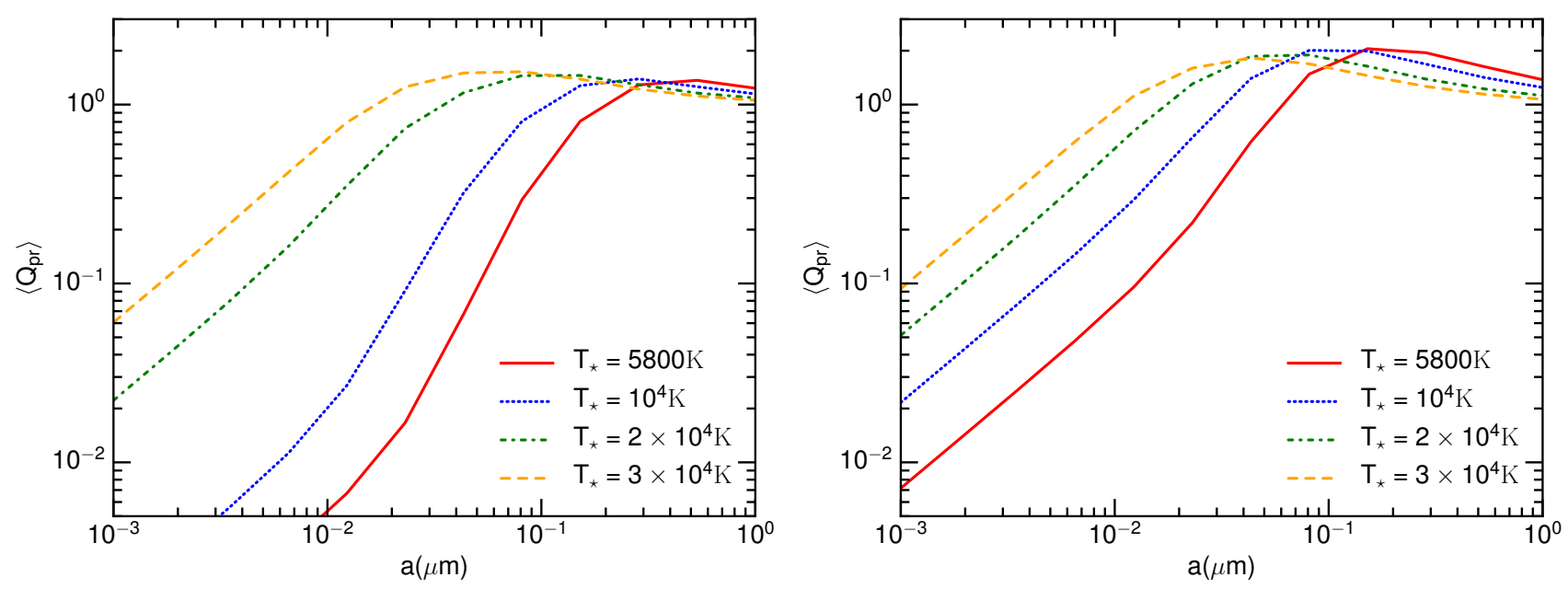

Figure 7. Averaged radiation pressure cross-section efficiency as a function of the grain size assuming the stellar radiation with $T_{\star}=5800 \mathrm{~K}, 10000 \mathrm{~K}, 20000,30000 \mathrm{~K}$ for silicate (left panel) and graphite (right panel). Rapid decrease of $\left\langle Q_{\mathrm{pr}}\right\rangle \propto a$ for $a<0.01 \mu \mathrm{m}$ for the solar radiation field.

The radiation pressure cross-section efficiency is given by

$$
Q_{\mathrm{pr}}(a, \lambda)=Q_{\mathrm{abs}}+Q_{\mathrm{sca}}(1-\cos \theta)
$$

where $\theta$ is the scattering angle. The average cross-section efficiency is obtained by integrating over the radiation spectrum (see Hoang et al. 2015):

$$
\left\langle Q_{\mathrm{pr}}(a)\right\rangle=\frac{\int Q_{\mathrm{pr}} u_{\lambda} d \lambda}{\left(\int u_{\lambda} d \lambda\right)}
$$

denotes the averaging over the spectrum of the solar radiation field of energy density $u_{\lambda}$.

We calculate the cross-sections for spherical grains using the Mie theory for astronomical silicate and graphite grains. Figure 7 shows $\left\langle Q_{\mathrm{pr}}\right\rangle$ as a function of the grain size for three stellar radiation field. For the solar radiations spectrum, $Q_{\mathrm{pr}} \sim 0.1$ for $a \sim 0.01 \mu \mathrm{m}$ and scales as $0.1(0.01 \mu \mathrm{m} / a)$ for smaller grains.

\section{B. OVERVIEW OF BASIC PHYSICS OF CIRCUMSOLAR DUST AND DUST-FREE-ZONE}

\section{B.1. Radiation force, gravity, and Poynting-Robertson drag}

Dust grains in the interplanetary medium are subject to various processes, including solar gravity, radiative pressure, molecular force by the solar wind, and Poynting-Robertson (P-R) drag. The radiative force on a spherical grain of size $a$ located at distance $R$ from the Sun is given by

$$
F_{\mathrm{rad}}=\frac{L_{\odot}}{4 \pi R^{2} c}\left\langle Q_{\mathrm{pr}}(a)\right\rangle \pi a^{2},
$$

where $L_{\odot}$ is the solar luminosity.

The ratio of radiative force to gravity force is given by

$$
\beta=-\frac{F_{\mathrm{rad}}}{F_{\mathrm{gra}}}=\frac{3 L_{\odot}\left\langle Q_{\mathrm{pr}}(a, \lambda)\right\rangle}{16 \pi G M_{\odot} c}(\rho a)^{-1} \approx 1.91\left(\frac{\left\langle Q_{\mathrm{pr}}(a)\right\rangle}{1.0}\right) \frac{1}{\hat{\rho} a_{-5}} .
$$

Grains orbiting the Sun experience Poyingting-Robertson drag. Burns et al. (1979) showed that the P-R force acts to gradually decrease the semimajor axis and eccentricity of the orbit of micron-sized grains around the Sun, resulting in the loss of micron-sized grains. The orbital decay time for a dust grain on circular orbit is given by

$$
t_{\mathrm{P}-\mathrm{R}}=\frac{R^{2}}{4 \eta\left\langle Q_{\mathrm{pr}}\right\rangle} \approx 7665 a_{-7} \hat{\rho} \frac{0.1}{\left\langle Q_{\mathrm{pr}}\right\rangle}\left(\frac{R}{\mathrm{AU}}\right)^{2} \text { days },
$$


where $\eta=F_{0} R_{0}^{2} \pi a^{2} / m c^{2}=2.53 \times 10^{11}(\rho a)^{-1}$ with $F_{0}$ being the solar flux at distance $R_{0}$ with $m=\rho 4 \pi a^{3} / 3$.

For classical grains $(a \sim 0.1-1 \mu \mathrm{m})$, one has $\left\langle Q_{p r}\right\rangle \sim 1$, and the P-R drag timescale is shorter for smaller grains. For nanoparticles $(a<0.01 \mu \mathrm{m})$ in the solar radiation field, the average radiation pressure decreases with the grain size as $\left\langle Q_{p r}\right\rangle \propto a$ (see Figure 7), thus the P-R effect become independent on $a$.

\section{B.2. Thermal sublimation and dust-free-zone}

\section{B.2.1. Thermal sublimation}

Grains near the sun are heated to high temperature by solar radiation such that grains can sublimate rapidly. Guhathakurta \& Draine (1989) investigated the sublimation of dust grains using detailed balance and derived the sublimation rate for a grain of radius $a$ :

$$
\frac{d a}{d t}=-n_{d}^{-1 / 3} \nu_{0} \exp \left(\frac{-B}{k_{\mathrm{B}} T_{d}}\right)
$$

where $n_{d} \sim 10^{22}-10^{23} \mathrm{~cm}^{-3}$ is the atomic number density of dust, $B$ is the sublimation energy per atom, $\nu_{0}=$ $2 \times 10^{15} \mathrm{~s}^{-1}$ and $B / k_{\mathrm{B}}=68100-20000 N^{-1 / 3} \mathrm{~K}$ for silicate grains, $\nu_{0}=2 \times 10^{14} \mathrm{~s}^{-1}$ and $B / k_{\mathrm{B}}=81200-20000 N^{-1 / 3} \mathrm{~K}$ for carbonaceous grains with $N$ being the total number of atoms of the grain (Guhathakurta \& Draine 1989; Waxman \& Draine 2000).

The sublimation time of a dust grain with size $a$ is defined as

$$
t_{\mathrm{sub}}\left(T_{d}\right)=-\frac{a}{d a / d t}=a n_{d}^{1 / 3} \nu_{0}^{-1} \exp \left(\frac{B}{k_{\mathrm{B}} T_{d}}\right)
$$

where $d a / d t$ from Equation (B6) has been used.

Plugging the numerical parameters into the above equation, we obtain

$$
t_{\mathrm{sub}}\left(T_{d}\right)=6.36 \times 10^{3} a_{-5} \exp \left[68100 \mathrm{~K}\left(\frac{1}{T_{d}}-\frac{1}{1800 \mathrm{~K}}\right)\right] \mathrm{s}
$$

for silicate grains, and

$$
t_{\mathrm{sub}}\left(T_{d}\right)=1.36 a_{-5} \exp \left[81200 \mathrm{~K}\left(\frac{1}{T_{d}}-\frac{1}{3000 \mathrm{~K}}\right)\right] \mathrm{s}
$$

for graphite grains.

\section{B.2.2. Dust-free-zone}

When thermal sublimation is faster than the P-R drag time, grains are rapidly sublimated before they can be dragged inward by P-R, producing a dust-free-zone.

The equilibrium temperature of grains irradiated by solar radiation can be approximately given by

$$
\begin{gathered}
T_{d}=16.4 a_{-5}^{-1 / 15} U^{1 / 6} \mathrm{~K} \simeq 1916 a_{-5}^{-1 / 15}\left(\frac{R}{R_{\odot}}\right)^{-1 / 3}, \\
T_{d}=22.3 a_{-5}^{-1 / 15} U^{1 / 6} \mathrm{~K} \simeq 2376 a_{-5}^{-1 / 15}\left(\frac{R}{R_{\odot}}\right)^{-1 / 3},
\end{gathered}
$$

for silicate and graphite, respectively. These approximate formulae are valid for $U \leq 10^{4}$ and $0.01<a<1 \mu \mathrm{m}$ (silicates) and $0.005<a<0.15 \mu \mathrm{m}$ (graphite). So, to calculate $T_{d}$ for a general $U$, we directly compute by solving Eq. as in Hoang et al. (2015).

One can estimate the sublimation distance by setting $T_{d} \equiv T_{\text {sub }}$. Thus, the sublimation distance is then a function of the grain size

$$
R_{\mathrm{sub}}=2\left(\frac{T_{\mathrm{sub}}}{1500 \mathrm{~K}}\right)^{3}\left(\frac{a}{0.1 \mu \mathrm{m}}\right)^{-1 / 5} R_{\odot}
$$

Equation B12 reveals that the radius of dust-free-zone $R_{\text {sub }}$ is between $3-4 R_{\odot}$ for $a \sim 0.01-0.1 \mu \mathrm{m}$. 
The criteria for efficient sublimation is defined with respect to the period of Kepler motion of the dust grain required for the grain of initial distance $r$ to fall to $R_{f}=R_{\odot}$ (Mukai \& Yamamoto 1979):

$$
t_{K}=\frac{\left(R / R_{\odot}\right)^{3 / 2}}{[G(1-\beta)]^{1 / 2}} \simeq 0.11\left(\frac{R}{R_{\odot}}\right)^{3 / 2} \frac{1}{[(1-\beta)]^{1 / 2}} \text { days }
$$

where $\beta=F_{\text {rad }} / F_{\text {gra }}$ is the ratio of radiative force to gravity force.

Mukai \& Yamamoto (1979) adopted a ratio of $t_{\mathrm{sub}} / t_{K} \sim 10^{2}-10^{3}$ to define the dust-free-zone which is determined by this distance corresponding to $t_{\mathrm{sub}} / t_{K} \sim 10^{2}-10^{3}$ or $t_{\mathrm{sub}} \sim 10-100$ days. Due to RP drag, dust grains are dragged inward and concentrates around the dust-free-zone boundary, producing the circumsolar ring. The region with $t_{\mathrm{sub}} / t_{K}<10^{2}-10^{3}$ or $t_{\mathrm{sub}} \sim 10-100$ days is essentially dust-free-zone.

\section{REVIEW OF RADIATIVE TORQUE DISRUPTION (RATD) MECHANISM}

\section{C.1. Rotation rate of irregular grains spun-up by radiative torques}

In this section, we briefly describe radiative torques (RATs) and spin-up by RATs for irregular grains for reference. A detailed description can be found in our previous works (Hoang et al. 2019; Hoang 2019).

Radiative torque (RAT) arising from the interaction of an anisotropic radiation field with an irregular grain is defined as

$$
\Gamma_{\lambda}=\pi a^{2} \gamma u_{\lambda}\left(\frac{\lambda}{2 \pi}\right) Q_{\Gamma}
$$

where $\gamma$ is the anisotropy degree of the radiation field, $Q_{\Gamma}$ is the RAT efficiency, and $a$ is the effective size of the grain which is defined as the radius of the sphere with the same volume as the irregular grain (Draine \& Weingartner 1996; Lazarian \& Hoang 2007a).

The magnitude of RAT efficiency, $Q_{\Gamma}$, can be approximated by a power-law (Hoang \& Lazarian 2008):

$$
Q_{\Gamma} \sim 0.4\left(\frac{\lambda}{1.8 a}\right)^{\eta}
$$

where $\eta=0$ for $\lambda \lesssim 1.8 a$ and $\eta=-3$ for $\lambda>1.8 a$.

Numerical calculations of RATs for several shapes of different optical constants in Lazarian \& Hoang (2007a) find the slight difference in RATs among the realization. An extensive study for a large number of irregular shapes by Herranen et al. (2019) shows little difference in RATs for silicate, carbonaceous, and iron compositions. Moreover, the analytical formula (Equation C15) is also in a good agreement with their numerical calculations. Therefore, one can use Equation (C15) for the different grain compositions and grain shapes, and the difference is an order of unity. We note that the above scaling (Eq. C15) is valid for grains of sizes $a<\lambda / 0.1$ only (Lazarian \& Hoang 2007a; Herranen et al. 2019). Calculations of RATs for larger grains of $a>\lambda / 0.1$ are not yet available due to the expensive computing time. However,

The average radiative torque efficiency over the spectrum is defined as

$$
\bar{Q}_{\Gamma}=\frac{\int \lambda Q_{\Gamma} u_{\lambda} d \lambda}{\int \lambda u_{\lambda} d \lambda}
$$

For interstellar grains with $a \lesssim a_{\text {trans }}=\bar{\lambda} / 1.8, \bar{Q}_{\Gamma}$ can be approximated to (Hoang \& Lazarian 2014)

$$
\bar{Q}_{\Gamma} \simeq 2\left(\frac{\bar{\lambda}}{a}\right)^{-2.7} \simeq 2.6 \times 10^{-2}\left(\frac{\bar{\lambda}}{0.5 \mu \mathrm{m}}\right)^{-2.7} a_{-5}^{2.7},
$$

and $\overline{Q_{\Gamma}} \sim 0.4$ for $a>a_{\text {trans }}$.

Therefore, the averaged radiative torque can be given by

$$
\begin{aligned}
\Gamma_{\mathrm{RAT}} & =\pi a^{2} \gamma u_{\mathrm{rad}}\left(\frac{\bar{\lambda}}{2 \pi}\right) \bar{Q}_{\Gamma} \\
& \simeq 5.8 \times 10^{-29} a_{-5}^{4.7} \gamma_{\mathrm{rad}} U \bar{\lambda}_{0.5}^{-1.7} \mathrm{erg}
\end{aligned}
$$


for $a \lesssim a_{\text {trans }}$, and

$$
\Gamma_{\mathrm{RAT}} \simeq 8.6 \times 10^{-28} a_{-5}^{2} \gamma U \bar{\lambda}_{0.5} \mathrm{erg},
$$

for $a>a_{\text {trans }}$, where $\bar{\lambda}_{0.5}=\bar{\lambda} / 0.5 \mu \mathrm{m}$

The well-known damping process for a rotating grain is sticking collisions with gas atoms, followed by thermal evaporation. Thus, for a gas with He of $10 \%$ abundance, the characteristic damping time is

$$
\begin{aligned}
\tau_{\text {gas }} & =\frac{3}{4 \sqrt{\pi}} \frac{I}{1.2 n_{\mathrm{H}} m_{\mathrm{H}} v_{\mathrm{th}} a^{4}} \\
& \simeq 8.74 \times 10^{4} a_{-5} \hat{\rho}\left(\frac{30 \mathrm{~cm}^{-3}}{n_{\mathrm{H}}}\right)\left(\frac{100 \mathrm{~K}}{T_{\text {gas }}}\right)^{1 / 2} \mathrm{yr},
\end{aligned}
$$

where $v_{\mathrm{th}}=\left(2 k_{\mathrm{B}} T_{\text {gas }} / m_{\mathrm{H}}\right)^{1 / 2}$ is the thermal velocity of a gas atom of mass $m_{\mathrm{H}}$ in a plasma with temperature $T_{\text {gas }}$ and density $n_{\mathrm{H}}$, the spherical grains are assumed (Hoang \& Lazarian 2009; Draine \& Weingartner 1996). This time is equal to the time required for the grain to collide with an amount of gas of the grain mass.

IR photons emitted by the grain carry away part of the grain's angular momentum, resulting in the damping of the grain rotation. For strong radiation fields or not very small sizes, grains can achieve equilibrium temperature, such that the IR damping coefficient (see Draine \& Lazarian 1998) can be calculated as

$$
F_{\mathrm{IR}} \simeq\left(\frac{0.4 U^{2 / 3}}{a_{-5}}\right)\left(\frac{30 \mathrm{~cm}^{-3}}{n_{\mathrm{H}}}\right)\left(\frac{100 \mathrm{~K}}{T_{\text {gas }}}\right)^{1 / 2}
$$

Other rotational damping processes include plasma drag, ion collisions, and electric dipole emission. These processes are mostly important for PAHs and very small grains (Draine \& Lazarian 1998; Hoang et al. 2010; Hoang et al. 2011). Thus, the total rotational damping rate for large grains by gas collisions and IR emission can be written as

$$
\tau_{\text {damp }}=\frac{\tau_{\text {gas }}}{1+F_{\mathrm{IR}}} .
$$

Due to intense solar radiation field of $U \gg 10^{10}$, the rotational damping by IR emission dominates over gas damping, i.e., $F_{\mathrm{IR}} \gg 1$. Therefore,

$$
\tau_{\text {damp }} \simeq 4.7 a_{-5}^{2}\left(\frac{U}{10^{7}}\right)^{-2 / 3} \mathrm{yr}
$$

which does not depend on the gas properties. At $r=0.1 \mathrm{AU}, U \sim 5 \times 10^{9}, \tau_{\text {damp }} \sim 27$ days for $a \sim 0.1 \mu \mathrm{m}$ grains.

For the solar radiation source with stable luminosity, radiative torques $\Gamma_{\mathrm{RAT}}$ is constant, and the grain velocity is steadily increased over time. The equilibrium rotation can be achieved at (see Lazarian \& Hoang 2007a; Hoang \& Lazarian 2009; Hoang \& Lazarian 2014):

$$
\omega_{\mathrm{RAT}}=\frac{\Gamma_{\mathrm{RAT}} \tau_{\mathrm{damp}}}{I}
$$

where $I=8 \pi \rho a^{5} / 15$ is the grain inertia moment.

\section{REFERENCES}

Abbas, M. M., Craven, P. D., Spann, J. F., et al. 2004, ApJ, 614, 781

Burke, J. R., \& Silk, J. 1974, ApJ, 190, 1

Burns, J. A., Lamy, P. L., \& Soter, S. 1979, Icarus, 40, 1

Dolginov, A. Z., \& Mitrofanov, I. G. 1976,

(Astronomicheskii Zhurnal, 19, 758
Draine, B. T., \& Flatau, P. J. 1994, Journal of the Optical Society of America A: Optic s and Image Science, 11, 1491

Draine, B. T., \& Flatau, P. J. 2004, eprint arXiv, 9262

Draine, B. T., \& Lazarian, A. 1998, ApJ, 508, 157

Draine, B. T., \& Weingartner, J. C. 1996, ApJ, 470, 551 
Fox, N. J., Velli, M. C., Bale, S. D., et al. 2016, Space Science Reviews, 204, 7

Gruen, H., Fechtig, H., \& Kissel, J. 1985, in IN: Properties and interactions of interplanetary dust; Proceedings of the Eighty-fifth Colloquium, Max-Planck-Institut für Kernphysik, Heidelberg, Germany, 105-111

Guhathakurta, P., \& Draine, B. T. 1989, ApJ, 345, 230

Herranen, J., Lazarian, A., \& Hoang, T. 2019, ApJ, 878, 96

Herranen, J., Lazarian, A., \& Hoang, T. 2021, ApJ, 913, 63

Hoang, T. 2019, ApJ, 876, 13

Hoang, T., Cho, J., \& Lazarian, A. 2018, ApJ, 852, 129

Hoang, T., Draine, B. T., \& Lazarian, A. 2010, ApJ, 715, 1462

Hoang, T., \& Lazarian, A. 2008, MNRAS, 388, 117

Hoang, T., \& Lazarian, A. 2009, ApJ, 695, 1457

Hoang, T., \& Lazarian, A. 2014, MNRAS, 438, 680

Hoang, T., \& Lazarian, A. 2016, ApJ, 831, 159

Hoang, T., Lazarian, A., \& Draine, B. T. 2011, ApJ, 741, 87

Hoang, T., Lazarian, A., \& Schlickeiser, R. 2015, ApJ, 806, 255

Hoang, T., \& Lee, H. 2020, ApJ, 896, 144

Hoang, T., \& Tram, L. N. 2020, ApJ, 891, 0

Hoang, T., Tram, L. N., Lee, H., \& Ahn, S.-H. 2019, NatAs, 3, 766

Howard, R. A., Vourlidas, A., Bothmer, V., et al. 2019, Nature, 576, 232

Ip, W.-H., Lai, I.-L., \& Shen, F. 2019, Journal of Physics: Conference Series, 1332, 012007

Jones, A. P., Tielens, A. G. G. M., Hollenbach, D. J., \& McKee, C. F. 1994, ApJ, 433, 797

Kimura, H., \& Mann, I. 1998, Earth, 50, 493

Kimura, H., Mann, I., Biesecker, D. A., \& Jessberger, E. K. 2002, Icarus, 159, 529

Kimura, H., Wada, K., Yoshida, F., et al. 2020, MNRAS, 496, 1667

Kobayashi, H., Kimura, H., Watanabe, S.-i., Yamamoto, T., \& Müller, S. 2012, Earth, Planets and Space, 63, 1067
Lamy, P., Kuhn, J. R., Lin, H., Koutchmy, S., \& Smartt, R. N. 1992, Science (ISSN 0036-8075), 257, 1377

Lamy, P. L. 1974, A\&A, 35, 197 , a\&AA ID. AAA012.106.023

Lazarian, A., \& Hoang, T. 2007a, MNRAS, 378, 910

Lazarian, A., \& Hoang, T. 2007b, ApJ, 669, L77

Lazarian, A., \& Hoang, T. 2019, ApJ, 883, 122

Lazarian, A., \& Hoang, T. 2021, ApJ, 908, 12

Leinert, C., Hanner, M., Link, H., \& Pitz, E. 1978, A\&A, 64,119

Mann, I. 1992, A\&A(ISSN 0004-6361), 261, 329

Mann, I. 2017, Philosophical Transactions of the Royal Society A: Mathematical, Physical and Engineering Sciences, 375, 20160254

Mann, I., Murad, E., \& Czechowski, A. 2007, Planetary and Space Science, 55, 1000

Mann, I., Kimura, H., Biesecker, D. A., et al. 2004, Space Science Reviews, 110, 269

Mathis, J. S., Mezger, P. G., \& Panagia, N. 1983, A\&A, 128,212

Misconi, N. Y. 1993, Journal of Geophysical Research (ISSN 0148-0227), 98, 18

Mukai, T., \& Schwehm, G. 1981, A\&A, 95, 373

Mukai, T., \& Yamamoto, T. 1979, Publications of the Astronomical Society of Japan, 31, 585

Paddack, S. J. 1969, Journal of Geophysical Research, 74, 4379

Paddack, S. J., \& Rhee, J. W. 1975, Geophysical Research Letters, 2, 365

Parker, E. N. 1958, ApJ, 128, 664

Russell, H. N. 1929, ApJ, 69, 49

Sanchez-Diaz, E., Rouillard, A. P., Lavraud, B., et al. 2016, Journal of Geophysical Research: Space Physics, 121, 2830

Tatsuuma, M., Kataoka, A., \& Tanaka, H. 2019, ApJ, 874, 159

Venzmer, M. S., \& Bothmer, V. 2018, A\&A, 611, A36

Waxman, E., \& Draine, B. T. 2000, ApJ, 537, 796 